\title{
bNEAT: a Bayesian network method for detecting epistatic interactions in genome-wide association studies
}

\author{
Bing Han, Xue-wen Chen ${ }^{*}$ \\ From IEEE International Conference on Bioinformatics and Biomedicine 2010 \\ Hong Kong, P. R. China. 18-21 December 2010
}

\begin{abstract}
Background: Detecting epistatic interactions plays a significant role in improving pathogenesis, prevention, diagnosis and treatment of complex human diseases. A recent study in automatic detection of epistatic interactions shows that Markov Blanket-based methods are capable of finding genetic variants strongly associated with common diseases and reducing false positives when the number of instances is large. Unfortunately, a typical dataset from genome-wide association studies consists of very limited number of examples, where current methods including Markov Blanket-based method may perform poorly.

Results: To address small sample problems, we propose a Bayesian network-based approach (bNEAT) to detect epistatic interactions. The proposed method also employs a Branch-and-Bound technique for learning. We apply the proposed method to simulated datasets based on four disease models and a real dataset. Experimental results show that our method outperforms Markov Blanket-based methods and other commonly-used methods, especially when the number of samples is small.

Conclusions: Our results show bNEAT can obtain a strong power regardless of the number of samples and is especially suitable for detecting epistatic interactions with slight or no marginal effects. The merits of the proposed approach lie in two aspects: a suitable score for Bayesian network structure learning that can reflect higher-order epistatic interactions and a heuristic Bayesian network structure learning method.
\end{abstract}

\section{Background}

Genome-wide association study (GWAS) focuses on studies of the genetic variants related with a variety of diseases from individual to individual among a cohort of cases (people with the disease) and controls (similar people without the disease) [1-3]. The most important category of genetic variations is SNP (Single Nucleotide Polymorphism), which influences disease risk. Conventional analysis methods for GWAS data only consider one SNP at a time by the Armitage trend test (ATT) and are likely to miss genetic variants having slight to

\footnotetext{
* Correspondence: xwchen@ku.edu

Bioinformatics and Computational Life Sciences Laboratory, ITTC, Department of Electrical Engineering and Computer Science, The University of Kansas, 1520 West 15th Street, Lawrence, KS 66045, USA

Full list of author information is available at the end of the article
}

moderate marginal effects but strong joint effects on disease risk. Moreover, it is widely acknowledged that some common complex diseases such as various types of cancers, cardiovascular disease, and diabetes are caused by multiple genetic variants [4]. Therefore, there is an urgent need to detect high-order epistasis (genegene interaction), which refers to the interactive effect of two or more genetic variants on complex human diseases, and explore how these epistatic interactions confer susceptibility to complex diseases [5]. However, the very large number of SNPs checked in a typical GWAS (more than 10 million) and the enormous number of possible SNP combinations make detecting high-order epistatic interactions from GWAS data statistically and computationally challenging $[6,7]$.

\section{Ciomed Central}


During the past decade, some heuristic computational methods have been proposed to detect causal interacting genes or SNPs. One type of computational methods for epistatic interactions detection are statistical methods including multifactor dimensionality reduction (MDR) [8-11], penalized logistic regression (stepPLR [12], lassoPLR [13]), and Bayesian epistasis association mapping (BEAM) methods [14]. MDR is a non-parametric and model-free method based on constructing a risk table for every SNP combination [11]. If the case and control ratio in a cell of this risk table is larger than 1, MDR will label it as "high risk", otherwise, "low risk". By the risk table, MDR can predict disease risk and will select the SNP combination with the highest prediction accuracy. StepPLR and lassoPLR make some modifications to avoid the overfitting problem of standard logistic regression when detecting epistatic interactions [15]. For example, stepPLR combines the LR criterion with a penalization of the L2-norm of the coefficients. This modification makes stepPLR more robust to high-order epistatic interactions [12]. In general, most statistical methods can only be applied to small-scale analysis (i.e., a small set of SNPs) due to their computational complexity. Moreover, MDR, stepPLR and lassoPLR are all predictor-based methods, which make them easy to include false positives. Comparing to MDR, stepPLR and lassoPLR, BEAM is a scalable and non-predictorbased statistical method [14]. BEAM partitions SNPs into three groups: group 0 is for normal SNPs, group 1 contains disease SNPs affecting disease risk independently, and group 2 contains disease SNPs that jointly contribute to the disease risk (interactions). Give a fixed partition, BEAM can get the posterior probability of this partition from SNP data based on Bayes theory. A Markov Chain Monte Carlo method is used to reach the optimal SNP partition with maximum posterior probability in BEAM. One drawback of BEAM is that identifying both single disease SNP and SNP combinations simultaneously make BEAM over-complex and weakens its power.

An alternative approach is machine learning based methods, which are based on binary classification (prediction) and treat cases as positives and controls as negatives in SNP data. Support vector machine-based approaches [16] and random forest-based approaches [17] are two commonly-used machine learning methods for epistatic interactions detection. They use SVM or random forest as a predictor and select a set of SNPs with the highest prediction/classification accuracy by feature selection. Like predictor-based statistical methods, machine learning-based methods lack the capability of detecting causal elements and tend to introduce many false positives, which may result in a huge cost for further biological validation experiments [18].
Recently, we propose a new Markov Blanket-based method, DASSO-MB, to detect epistatic interactions in case-control studies [18]. The Markov Blanket is a minimal set of variables, which can completely shield the target variable from all other variables based on Markov condition property. Thus, DASSO-MB can detect the SNP set that shows a strong association with diseases with the fewest false positives. Furthermore, the heuristic search strategy in DASSO-MB can avoid the timeconsuming training process as in SVMs and Random Forests.

In this paper, we address the problems by introducing a Bayesian networks-based method, which also employs a Branch-and-Bound technique to detect epistatic interactions. Bayesian networks provide a succinct representation of the joint probability distribution and conditional independence among a set of variables. In general, a structure learning methods for Bayesian networks first defines a score reflecting the fitness between each possible structure and the observed data, and then searches for a structure with the maximum score. Comparing to Markov Blanket based methods, the merits of applying Bayesian networks method to epistatic interaction detection includes: (1) BDE, BIC or MDL scores for Bayesian network structure learning can reflect higherorder interactions and are not sample-consuming; and (2) heuristic Bayesian network structure learning method can solve the classical XOR problem, which may hinder the applications of Markov blanket based approaches.

We apply the bNEAT (Bayesian Networks based Epistatic Association sTudies) method to simulated datasets based on four disease models and a real dataset (the Age-related Macular Degeneration (AMD) dataset). We demonstrate that the proposed method outperforms Markov Blanket methods and other commonly-used methods, especially when the number of samples is small.

\section{Results}

\section{Analysis of simulation data}

We first evaluate the proposed bNEAT method on simulated data sets, which are generated from three commonly used two-locus epistatic models in [15] and one three-locus epistatic model developed in [14]. Model-1 is a multiplicative model, model-2 demonstrates two-locus interaction multiplicative effects and model-3 specifies two-locus interaction threshold effects. There are three disease loci in model-4 [14]. Some certain genotype combinations can increase disease risk in model-4 and there are almost no marginal effects for each disease locus.

To compare the performance of different methods, we use the same data generation process and the similar 
parameter settings as in $[14,15,18]$. We generate 50 datasets and each contains 100 markers genotyped for 1,000 cases and 1,000 controls. To measure the performance of each method, we use "power" as the criterion function. Power is calculated as follows:

$$
\text { Power }=\frac{N_{D}}{N}
$$

where $N$ is the total number of simulated datasets and $N_{D}$ is the number of simulated datasets in which all disease associated markers are identified without any false positives.

We compare the bNEAT algorithm with four methods: BEAM, Support Vector Machine, MDR and DASSO-MB on the four simulated disease models. The BEAM software is downloaded from http://www.fas.harvard.edu/ junliu/BEAM and we set the threshold of the $B$ statistic as 0.1 [14]. For support vector machines, we use LIBSVM with a RBF kernel to detect gene-gene interactions and the detail is shown in [18]. Since MDR algorithm can not be applied to a large dataset directly, we first reduce the number of SNPs to 10 by ReliefF [19], a commonly-used feature selection algorithm, and then MDR performs an exhaustive search for a SNP set that can maximize cross-validation consistency and prediction accuracy. For DASSO-MB, we set the threshold of $G^{2}$ test as 0.01 to determine (conditional) dependence and (conditional) independence.

The results on the simulated data are shown in Figures 1 and 2. As can be seen, among the five methods, the bNEAT algorithm performs the best. BEAM is worse than both bNEAT and DASSO-MB. One possible reason is that BEAM tries to detect single disease loci and epistatic interactions simultaneously. This strategy is unnecessary and makes BEAM over-complex. The other possible reason is that BEAM uses fixed Dirichlet priors in its Bayesian marker partition model, which may not reflect and penalize the model complexity appropriately [20].

Typically, GWAS can not generate a large number of samples due to the high experiment cost. Thus, the performance of various computational methods for epistatic interaction detection in case of small samples is important. We explore the effect of the number of samples on the performance of bNEAT, DASSO-MB, BEAM and SVM. We generate synthetic datasets containing 40 markers genotyped for different number of cases and controls with $r^{2}=1$ and MAF $=0.5$.

The results are shown in Figure 3. We find that bNEAT is more sample-efficient than other methods in that it can achieve the highest power when the number of samples is the same. In addition, it needs fewer samples to reach the perfect power comparing to other

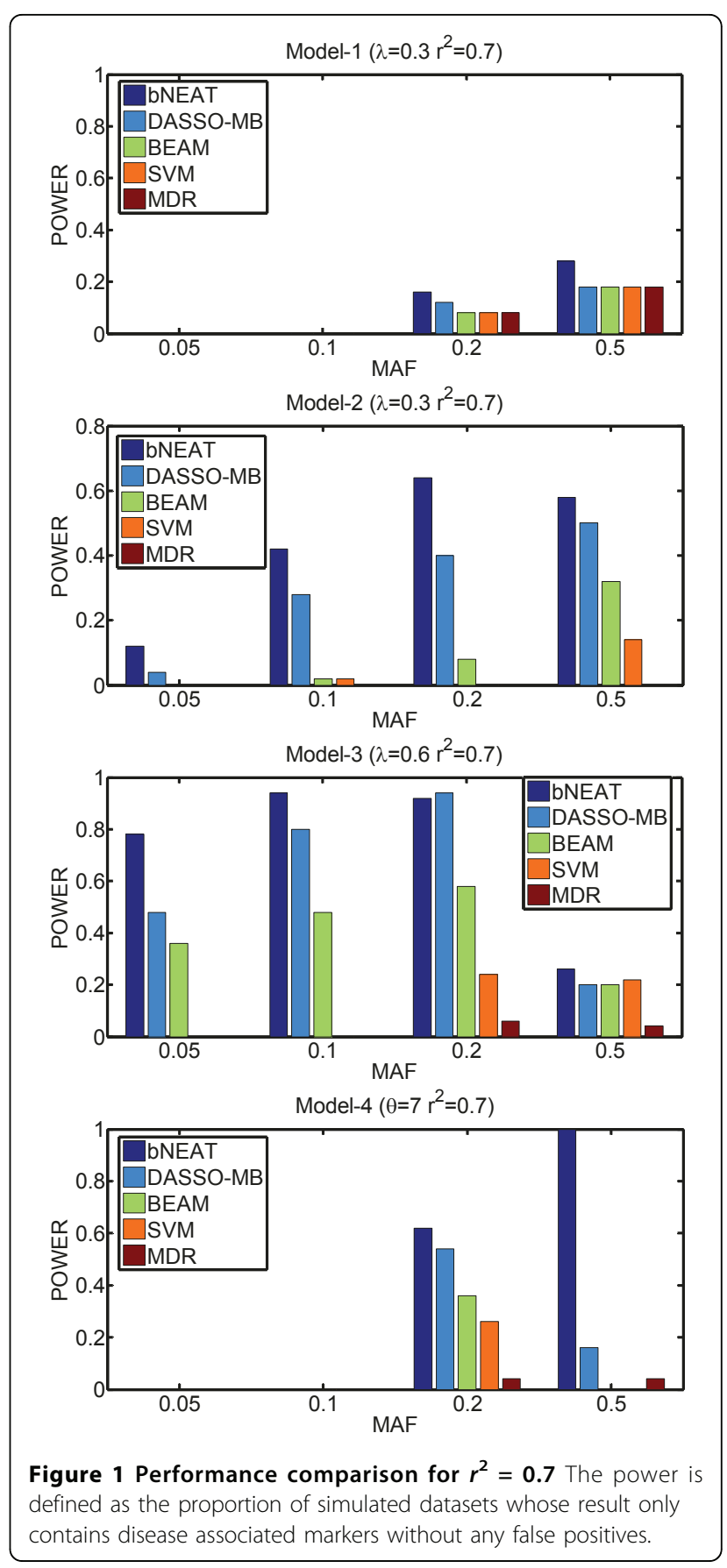

methods. DASSO-MB is the second best. For models 13 , almost all methods can obtain a perfect power except SVM when the number of samples is larger than 4000. SVM can not achieve a perfect power even though we have sufficient samples $(\geq 8000)$. This may indicate that the predictor-based methods lack the ability to find causal elements precisely. The result from model- 4 is particularly interesting: bNEAT exhibits overwhelming superiority over other three methods, as bNEAT yields a 


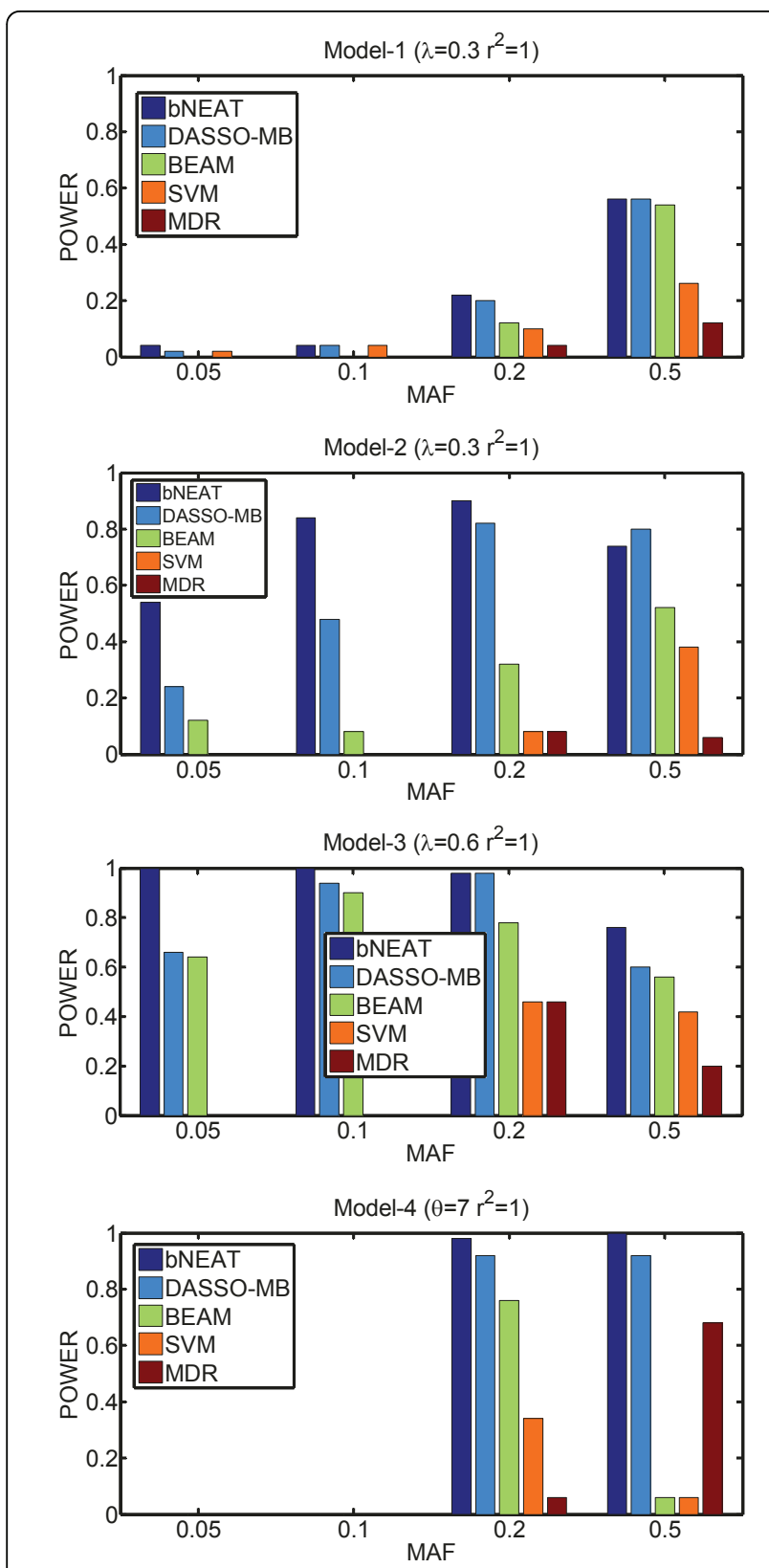

Figure 2 Performance comparison for $r^{2}=1$ The power is defined as the proportion of simulated datasets whose result only contains disease associated markers without any false positives.

perfect power even the number of samples is small (around 400), which indicates that bNEAT is especially suitable for detecting epistatic interactions with slight or no marginal effects.

\section{Results on AMD data}

In this section, we apply bNEAT to large-scale (large number of SNPs but small samples) datasets in real genome-wide case-control studies, which often require

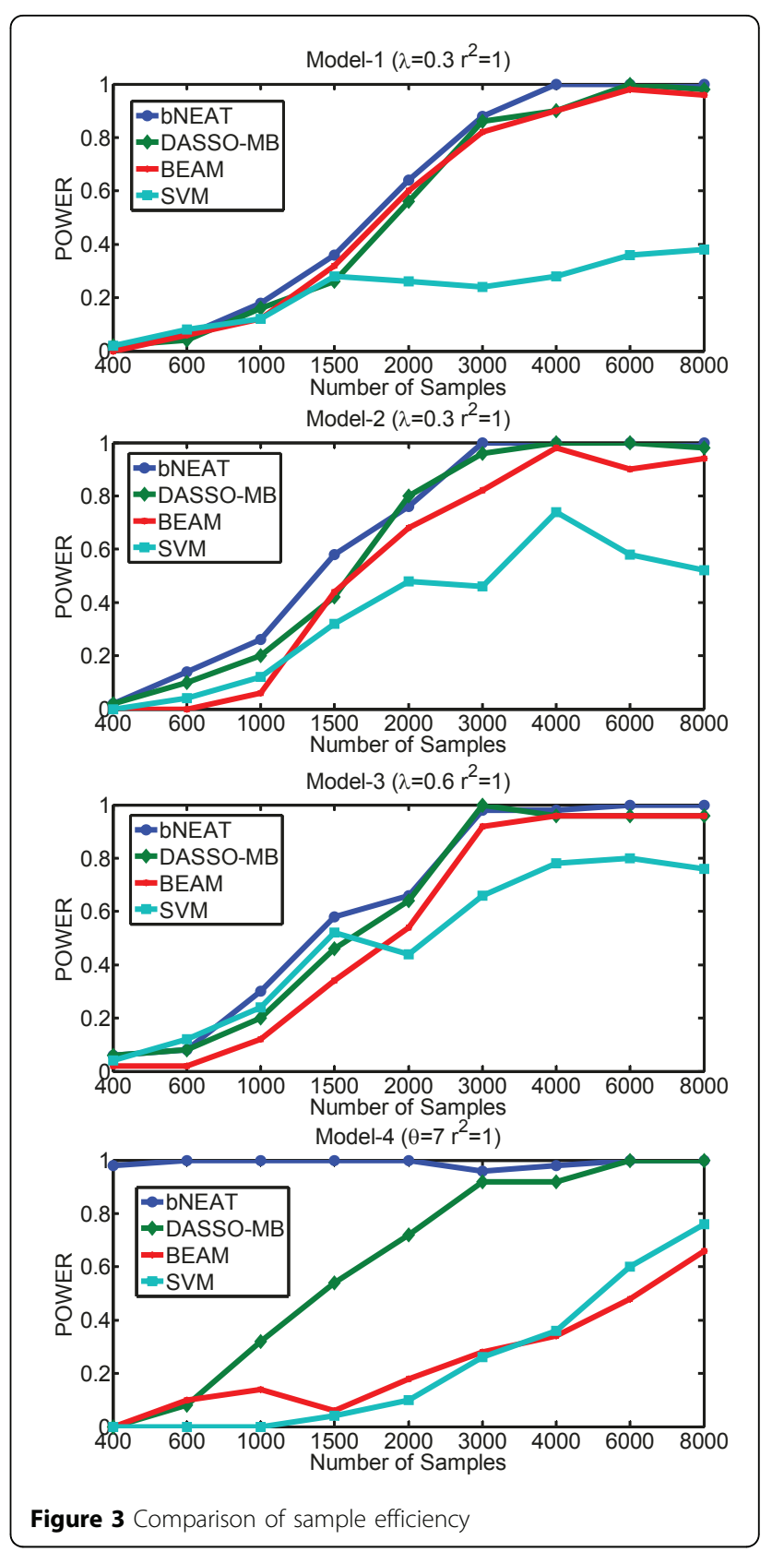

genotyping of 30,000-1,000,000 common SNPs. We make use of an Age-related Macular Degeneration (AMD) dataset containing 116,204 SNPs genotyped with 96 cases and 50 controls [21]. Multiple genetic factors cause AMD, which can result in a loss of vision.

To remove inconsistently genotyped SNPs, we perform filtering process as in [18]. After filtering, there are 97,327 SNPs remained. Since the number of SNPs is very large, restricting the search space to avoid unreasonable search by selecting some candidate SNPs as in [22] is necessary. We select top 200 candidate SNPs based on $G^{2}$ test and then use bNEAT to identify 
disease SNPs related with AMD. bNEAT detects three associated SNPs: rs380390, rs3913094 and rs10518433. The first SNP, rs380390, is already found in [21] with a significant association with AMD. Although no evidences were reported with the other two SNPs related to AMD in the literature, they may be plausible candidate SNPs associated with AMD.

\section{Conclusions and discussion}

Comparing with many computational methods used for identification of epistatic interactions, Markov Blanket based method can increase power and reduce false positives. However, Markov Blanket based method is sample-consuming and the greedy searching strategy in Markov Blanket method is not suitable for detecting some interaction models with no independent main effects for each disease locus. In this paper, we propose a Bayesian networks method based on Branch-andBound technique (bNEAT) to detect epistatic interactions. We demonstrate that the proposed bNEAT method significantly outperforms Markov Blanket method and other commonly-used methods, especially when the number of samples is small.

Even though the bNEAT method is more powerful than Markov Blanket based method, it can not be directly applied to genome-wide dataset due to the large number of SNPs. Integrating Markov chain Monte Carlo or simulated annealing technique into our bNEAT method to make it scalable to genome-wide dataset is one direction for future research. Moreover, we will explore different score schemes for epistatic interaction detection by Bayesian networks. For example, information-based score schemes (e.g., AIC score and BIC score) are derived in case of large number of samples [23]. When the number of samples is small, the approximation in the inference of both AIC score and BIC score can not hold any more. In fact, the penalty term for model complexity in AIC score and BIC score can also reflect the variance of the model [24]. Thus in our future work, we will design a new score scheme by estimating the penalty term from data to make sure that the score scheme can fit data better.

\section{Methods}

\section{Bayesian networks}

A Bayesian network is a directed acyclic graph (DAG) $G$ consisting of nodes corresponding to a random variable set $X=\left\{X_{1}, X_{2}, \ldots, X_{n}\right\}$ and edges between nodes, which determine the structure of $G$ and therefore the joint probability distribution of the whole network [25].

Definition 1 (Conditional Independence)For three random variables (nodes) $X, Y$ and $Z$, if the probability distribution of $X$ conditioned on both $Y$ and $Z$ is equal to the probability distribution of $X$ conditioned only on $Y$, i.e., $P(X \mid Y, Z)=P(X \mid Y), X$ is conditionally independent of $Z$ given $Y$.

This conditional independence is represented as . Similarly, represents conditional dependence [26].

Theorem 1 (Local Markov Assumption)Each variable is conditionally independent of its nondescendants, given its parents in the DAG G.

By applying the local Markov assumption, the joint probability distribution $J$ can be represented as

$$
P\left(X_{1}, \ldots, X_{n}\right)=\prod_{i=1}^{n} P\left(X_{i} \mid \operatorname{Pa}\left(X_{i}\right)\right)
$$

where $\mathrm{Pa}\left(X_{i}\right)$ denotes the set of parents of $X_{i}$ in $G$. Therefore, there are two components in a Bayesian network. The first component is the DAG G reflecting the structure of the network. The second component, $\theta$, describes the conditional probability distribution $P\left(X_{i} \mid\right.$ $\left.\mathrm{Pa}\left(X_{i}\right)\right)$ to specify the unique distribution $J$ on $G$.

Definition 2 (V-structure)For three nodes $X, Y$ and $Z$ in a Bayesian network, a structure with the form of $X \rightarrow Z \Re Y$ (no edge between $X$ and $Y$ ) is called a $\nu$-structure.

Definition 3 (D-seperation)For three nodes $X, Y$ and $Z$ in a Bayesian network, if there is no active path between $X$ and $Y$ given $Z$, we say that $X$ and $Y$ are $d$-seperated given $Z$, denoted as $\operatorname{Dsep}(X ; Y \mid Z)$.

Bayesian networks allow us to explore causal relationships to perform explanatory analysis and make predictions. As shown in Figure 4, GWAS attempts to identify the $k$-way interaction among SNPs: $\mathrm{SNP}_{1}$, $\mathrm{SNP}_{2}, \ldots, \mathrm{SNP}_{\mathrm{k}}$, which are associated with a disease. The $n$ SNP nodes and the disease status/label node construct a Bayesian network and we want to determine which SNP nodes are the parent nodes of the disease status /label node.

\section{Structure learning of Bayesian networks}

Even though a Bayesian network can be constructed by an expert, most tasks of determining the network structure are too complex for humans. We have no choice but to learn the network structure and parameters from data. There are two types of structure learning methods for Bayesian networks: constraint-based methods and score-and-search methods.

The constraint-based methods first build the skeleton of the network (undirected graph) by a set of dependence and independence relationships. Next constraintbased methods direct links in the undirected graph to construct a directed graph with $\mathrm{d}$-separation properties corresponding to the dependence and independence determined [27-29]. Even though constraint-based 


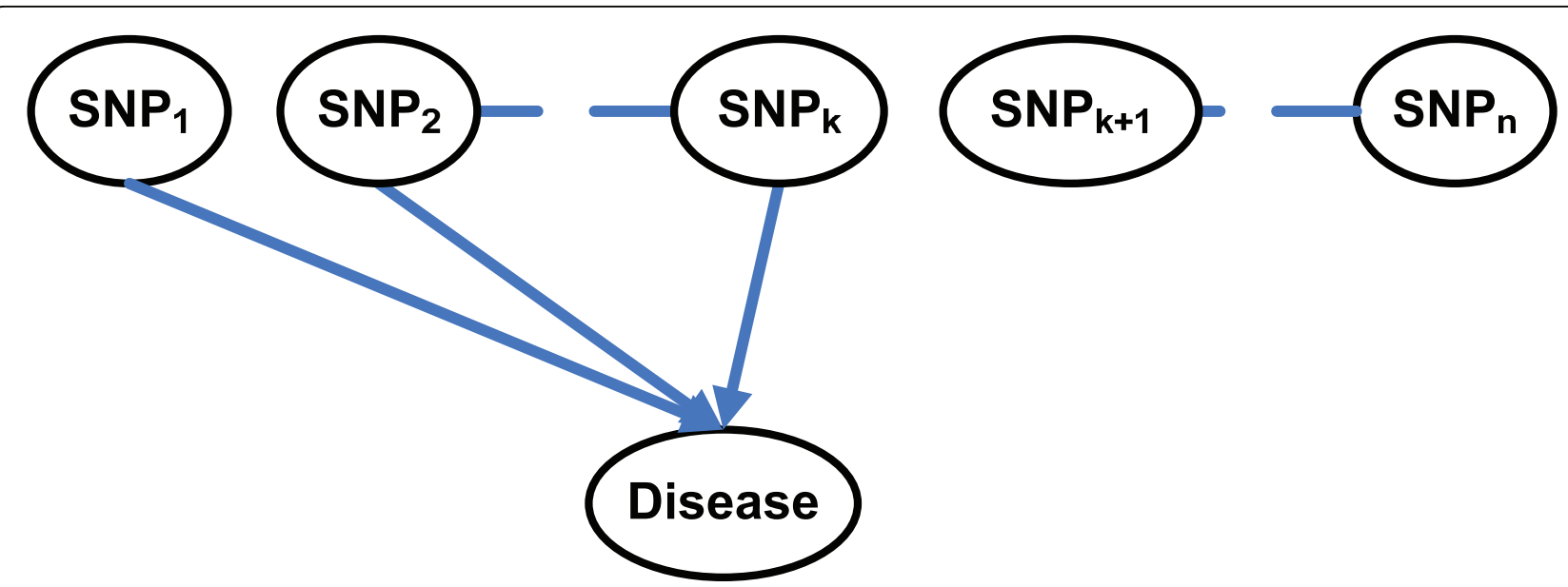

Figure 4 An Example of Genome-wide Association Studies. The goal of genome-wide association studies is to identify the k-way interaction among SNPs: $\mathrm{SNP}_{1}, \mathrm{SNP}_{2}, \ldots, \mathrm{SNP}_{\mathrm{k}}$, which are associated with disease.

methods are developed with a rigorous theoretical foundation, errors in conditional dependence and independence will affect the stability of constraint-based methods and this problem is especially serious when the number of samples is small.

The score-and-search methods view a Bayesian network as a statistical model and transform the structure learning of Bayesian network into a model selection problem [30]. To select the best model, a score function is needed to indicate the fitness between a network and the data. Then the learning task is to find the network with the highest score. Thus, score-and-search methods typically consist of two components, (1) a score function, and (2) a search procedure. In this paper, we focus on structure learning approaches for Bayesian networks based on score-and-search methods because score-andsearch methods are more robust for small data sets than constraint-based methods.

One of the most important issues in score-and-search methods is the selection of score function. A natural choice of score function is the likelihood function. However, the maximum likelihood score often overfits the data because it does not reflect the model complexity. Therefore, a good score function for Bayesian networks' structure learning must have the capability of balancing between the fitness and the complexity of a selected structure. There are several existing score functions based on a variety of principles, such as the information theory and minimum description length (BIC score, AIC score, MDL score) [31-33] and Bayesian approach (BDe score) [34].

The general idea of BDe score is to compute the posterior probability distribution. Consider that we want to learn the structure $S$ of a Bayesian network containing $n$ nodes from a dataset $D$ with $N$ examples, and let $q_{i}$ denote the number of configurations of the parent set $\mathrm{Pa}\left(X_{i}\right)$ of $X_{i}$ and let $r_{i}$ represent the number of states of $X_{i}$, the BDe score is obtained as

$$
P(S \mid D)=\prod_{i=1}^{n} \prod_{j=1}^{q_{i}} \frac{\Gamma\left(a_{i j}\right)}{\Gamma\left(a_{i j}+N_{i j}\right)} \prod_{k=1}^{r_{i}} \frac{\Gamma\left(a_{i j k}+N_{i j k}\right)}{\Gamma\left(a_{i j k}\right)}
$$

where $N_{i j k}$ is the number of cases for $X_{i}$ in its $k$ th con-

figuration and $\mathrm{Pa}\left(X_{i}\right)$ in the $j$ th configuration and $N_{i j}=\sum_{k=1}^{r_{i}} N_{i j k}$ priors which reflect a user's prior knowledge and we often set $a_{i j k}=N /\left(r_{i} q_{i}\right)$. BDe score can penalize the structure complexity inherently by integrating $P\left(D \mid \theta_{S}, S\right)$ and measuring the average expected likelihood over different possible choices of $\hat{\theta}_{S}\left(\hat{\theta}_{S}\right.$ is an estimate of parameters from the maximum likelihood method for the structure $S$ ) $[35,36]$. For AIC (Akaike information criterion) score and BIC (Bayesian information criterion) score, we can write a general score scheme as:

$$
\begin{aligned}
& \operatorname{Score}(S \mid D)=\log P\left(D \mid \theta_{S}, S\right)-C(S) f(N) \\
& C(S)=\sum_{i=1}^{n} q_{i}\left(r_{i}-1\right)
\end{aligned}
$$

where $C(S)$ represents the structure complexity [32] . The first term of this score scheme measures the fitness 
between the structure, and data and the second term reflects structure complexity. With a maximum likelihood method, we can get

$$
P\left(D \mid \theta_{S}, S\right)=\sum_{i=1}^{n} \sum_{j=1}^{q_{i}} \sum_{k=1}^{r_{i}} N_{i j k} \log \left(N_{i j k} / N_{i j}\right)
$$

In (4), by setting $f(N)=1$, we get the AIC score as

$$
\operatorname{AIC}(S \mid D)=\log P\left(D \mid \theta_{S}, S\right)-C(S)
$$

If we set $f(N)=1 / 2 \log (\mathrm{N})$, we get the BIC score, which is

$$
B I C(S \mid D)=\log P\left(D \mid \theta_{S}, S\right)-\frac{1}{2} C(S) \log (N)
$$

The BIC score are derived from a Taylor expansion and Laplace approximation when the number of samples $N$ approaches $\infty$. This results in a problem that the structure penalty term in (8) is very strict when the number of samples is small; therefore, we adjust the coefficient of the second term in (8) from $1 / 2$ to a smaller number (in our applications, we empirically set it to be 0.17 for all the datasets we study).

The computational task in score-and-search methods is to find a network structure with the highest score. The searching space consists of a superexponential number of structures- $2^{O\left(n^{2}\right)}$ and thus exhaustively searching optimal structure from data for Bayesian networks is NP-hard [37]. One simple heuristic search algorithm is greedy hill-climbing algorithm. In greedy hill-climbing algorithm, there are three types of operators that change one edge at each step:

- Add an edge

- Remove an edge

- Reverse an edge

By these three operators, we can construct the local neighbourhood of current network. Then we select the network with the highest score in the local neighbourhood to get the maximal gain. This process can be repeated until it reaches a local maximum. However, greedy hill-climbing algorithm cannot guarantee a global maximum [30]. Other structure learning methods for Bayesian networks include Branch-and-Bound (B\&B) [38,39], genetic algorithms [40] and Markov chain Monte Carlo [41]. Branch-and-Bound algorithms guarantee the optimal results in a significantly reduced search time compared to exhaustive search. Thus, we will employ B\&B algorithms in our study.

The proposed method uses $B \& B$ to search a structure that maximizes the BIC score. The algorithm is shown in Figure 5. bNEAT starts from an empty node set and

\section{Algorithm bNEAT}

INPUT: Data $D$, Disease label node, all $n$ SNP nodes

OUTPUT: Disease SNP nodes, which has the maximum BIC socre on Disease label node

Procedure $\left[\begin{array}{ll}S_{1} & P_{1}\end{array}\right]=\operatorname{bNEAT}\left(V_{1}\right)$ : Input: node set $V_{1}$. Output: BIC score $S_{1}$, parent set $P_{1}$.

\section{Begin}

1. Compute BIC score temp $S_{1}$ for $V_{1}$, $S_{1}=$ temp $S_{1}, \quad P_{1}=V_{1}$

2. IF $V_{1}=$ null then $\mathrm{i}=0$ else $\mathrm{i}=V_{1}$ (end)

3. For $i+1 \leq q \leq n$

\section{Begin}

(1) $V_{2}=V_{1} \cup q$ Compute BIC score temp $S_{2}$ for $V_{2}$

(2) IF temp $S_{2}>$ temp $S_{1}$ then $\left[\begin{array}{ll}S_{2} & P_{2}\end{array}\right]=$ bNEAT $\left(V_{2}\right)$

(3) IF $S_{2}>S_{1}$ then $S_{1}=S_{2}, \quad P_{1}=P_{2}$

End

\section{End}

Figure 5 the bNEAT algorithm

constructs a depth-first search tree to find the optimal parent (disease SNPs) set for the disease label node. In our B\&B search, instead of using the pruning strategy as in $[38,39]$, which sets a lower bound for the MDL score to prune the search tree, we stop the recursive calls when we observe that the BIC score will decrease on the children state of the current state. This strategy cannot guarantee global optima theoretically. However, it will significantly speed up the search process.

\section{Acknowledgements}

This work is supported by the US National Science Foundation Award IIS0644366.

This article has been published as part of BMC Genomics Volume 12 Supplement 2, 2011: Selected articles from the IEEE International Conference on Bioinformatics and Biomedicine 2010. The full contents of the supplement are available online at http://www.biomedcentral.com/14712164/12?issue=S2 


\section{Authors' contributions}

$\mathrm{BH}$ designed and implemented the algorithm. XWC conceived the study and designed the experiments. Both authors drafted the manuscript and approved the final manuscript.

\section{Competing interests}

Authors declare that they have no competing interests.

Published: 27 July 2011

\section{References}

1. Hirschhorn JN, Daly MJ: Genome-wide association studies for common diseases and complex traits. Nat Rev Genet 2005, 6:95-108

2. McCarthy Ml, Abecasis GR, Cardon LR, Goldstein DB, Little J, loannidis JP, Hirschhorn JN: Genome-wide association studies for complex traits: consensus, uncertainty and challenges. Nat Rev Genet 2008, 9:356-369.

3. Wang WY, Barratt BJ, Clayton DG, Todd JA: Genome-wide association studies: theoretical and practical concerns. Nat Rev Genet 2005, 6:109-118.

4. Cordell HJ: Detecting gene-gene interactions that underlie human diseases. Nat Rev Genet 2009, 10:392-404.

5. Cordell HJ: Epistasis: what it means, what it doesn't mean, and statistical methods to detect it in humans. Hum Mol Genet 2002, 11:2463-2468.

6. McKinney BA, Reif DM, Ritchie MD, Moore JH: Machine learning for detecting gene-gene interactions: a review. Appl Bioinformatics 2006, 5:77-88.

7. Musani SK, Shriner D, Liu N, Feng R, Coffey CS, Yi N, Tiwari HK, Allison DB: Detection of gene $x$ gene interactions in genome-wide association studies of human population data. Hum Hered 2007, 63:67-84.

8. Hahn LW, Ritchie MD, Moore $\mathrm{JH}$ : Multifactor dimensionality reduction software for detecting gene-gene and gene-environment interactions. Bioinformatics (Oxford, England) 2003, 19:376-382.

9. Moore JH, Gilbert JC, Tsai CT, Chiang FT, Holden T, Barney N, White BC: A flexible computational framework for detecting, characterizing, and interpreting statistical patterns of epistasis in genetic studies of human disease susceptibility. Journal of theoretical biology 2006, 241:252-261.

10. Ritchie MD, Hahn LW, Moore JH: Power of multifactor dimensionality reduction for detecting gene-gene interactions in the presence of genotyping error, missing data, phenocopy, and genetic heterogeneity. Genetic epidemiology 2003, 24:150-157.

11. Ritchie MD, Hahn LW, Roodi N, Bailey LR, Dupont WD, Parl FF, Moore JH: Multifactor-dimensionality reduction reveals high-order interactions among estrogen-metabolism genes in sporadic breast cancer. American journal of human genetics 2001, 69:138-147.

12. Park MY, Hastie T: Penalized logistic regression for detecting gene interactions. Biostatistics (Oxford, England) 2008, 9:30-50.

13. Wu $\Pi$, Chen YF, Hastie $T$, Sobel $E$, Lange K: Genome-wide association analysis by lasso penalized logistic regression. Bioinformatics (Oxford, England) 2009, 25:714-721.

14. Zhang Y, Liu JS: Bayesian inference of epistatic interactions in casecontrol studies. Nature genetics 2007, 39:1167-1173.

15. Marchini J, Donnelly P, Cardon LR: Genome-wide strategies for detecting multiple loci that influence complex diseases. Nature genetics 2005, 37:413-417.

16. Chen SH, Sun J, Dimitrov L, Turner AR, Adams TS, Meyers DA, Chang BL, Zheng SL, Gronberg H, Xu J, Hsu FC: A support vector machine approach for detecting gene-gene interaction. Genetic epidemiology 2008, 32:152-167.

17. Jiang $R$, Tang $W$, Wu $X, F u$ W: A random forest approach to the detection of epistatic interactions in case-control studies. BMC bioinformatics 2009, 10(Suppl 1):S65.

18. Han B, Park M, Chen XW: A Markov blanket-based method for detecting causal SNPs in GWAS. BMC bioinformatics 2010, 11(Suppl 3):S5.

19. Robnik-Šikonja M, Kononenko I: Theoretical and empirical analysis of ReliefF and RReliefF. Machine learning 2003, 53:23-69.

20. Ueno M: Learning networks determined by the ratio of prior and data. In Proceedings of 26th Conference Conference on Uncertainty in Artificial Intelligence: 8-11 July 2010; Corvallis, Oregon. Arlington: AUAI Press;P. Grünwald and P. Spirtes 2010:598-605

21. Klein RJ, Zeiss C, Chew EY, Tsai JY, Sackler RS, Haynes C, Henning AK, SanGiovanni JP, Mane SM, Mayne ST, et al: Complement factor H polymorphism in age-related macular degeneration. Science (New York, NY) 2005, 308:385-389.

22. Friedman N, Nachman I, Pe'er D: Learning Bayesian Network Structure from Massive Datasets: The "Sparse Candidate" Algorithm. In Proceedings of 15th Conference Conference on Uncertainty in Artificial Intelligence: 30 July -1August 1999; Stockholm, Sweden. San Fransisco:Morgan Kaufmann;Kathryn B. Laskey and Henri Prade 1999:206-215.

23. Burnham KP, Anderson DR, Hussong Fund M.Hazel: Model selection and multimodel inference : a practical information-theoretic approach. New York: Springer; 22002

24. Hastie T, Tibshirani R, Friedman JH: The elements of statistical learning : data mining, inference, and prediction. New York: Springer; 2001.

25. Chen XW, Anantha G, Wang X: An effective structure learning method for constructing gene networks. Bioinformatics (Oxford, England) 2006 22:1367-1374.

26. Chen X-W, Anantha G, Lin X: Improving Bayesian Network Structure Learning with Mutual Information-Based Node Ordering in the K2 Algorithm. IEEE Trans on Knowl and Data Eng 2008, 20:628-640.

27. Cheng J, Greiner R, Kelly J, Bell D, Liu W: Learning Bayesian networks from data: an information-theory based approach. Artif Intell 2002, 137:43-90.

28. Pearl J: Causality : models, reasoning, and inference. Cambridge, U.K. ; New York: Cambridge University Press; 22009.

29. Spirtes P, Glymour CN, Scheines R: Causation, prediction, and search Cambridge, Mass.: MIT Press; 22000.

30. Heckerman D, Geiger D, Chickering DM: Learning Bayesian Networks: The Combination of Knowledge and Statistical Data. Mach Learn 1995 20:197-243.

31. Akaike $\mathrm{H}$ : A new look at the statistical model identification. IEEE Transactions on Automatic Control 1974, 19:716-723.

32. Schwarz G: Estimating the dimension of a model. The Annals of Statistics $1978,6: 461-464$

33. Rissanen J: Stochastic Complexity and Modeling. The Annals of Statistics 1986, 14:1080-1100.

34. Cooper GF, Herskovits E: A Bayesian Method for the Induction of Probabilistic Networks from Data. Mach Learn 1992, 9:309-347.

35. Koller D, Friedman N: Probabilistic graphical models : principles and techniques. Cambridge, Mass.: MIT Press; 2009

36. Yu J, Smith VA, Wang PP, Hartemink AJ, Jarvis ED: Advances to Bayesian network inference for generating causal networks from observational biological data. Bioinformatics (Oxford, England) 2004, 20:3594-3603.

37. Chickering DM, Heckerman D, Meek C: Large-Sample Learning of Bayesian Networks is NP-Hard. J Mach Learn Res 2004, 5:1287-1330.

38. Suzuki J: Learning Bayesian Belief Networks Based on the Minimum Description Length Principle: An Efficient Algorithm Using the B \& B Technique. In Proceedings of 13th conference on machine learning: 3-6 July 1996; Bari, Italy. San Fransisco: Morgan Kaufmann;Lorenza Saitta 1996:462-470.

39. Tian J: A Branch-and-Bound Algorithm for MDL Learning Bayesian Networks. In Proceedings of 16th Conference Conference on Uncertainty in Artificial Intelligence: 30 June - 3 July 2000; Stanford, California. San Fransisco: Morgan Kaufmann;Craig Boutilier and Moisés Goldszmidt 2000:580-588.

40. Wong ML, Lam W, Leung KS: Using Evolutionary Programming and Minimum Description Length Principle for Data Mining of Bayesian Networks. IEEE Trans Pattern Anal Mach Intell 1999, 21:174-178.

41. Giudici P, Castelo R: Improving Markov Chain Monte Carlo Model Search for Data Mining. Machine learning 2003, 50:127-158.

doi:10.1186/1471-2164-12-S2-S9

Cite this article as: Han and Chen: bNEAT: a Bayesian network method for detecting epistatic interactions in genome-wide association studies. BMC Genomics 2011 12(Suppl 2):S9. 